\title{
ECG Biometrics Using Spectrograms and Deep Neural Networks
}

\author{
Nuno Bento, David Belo, and Hugo Gamboa
}

\begin{abstract}
The Electrocardiogram (ECG) is considered as a physiological signature and has previously been used for biometric purposes. The contamination of the signal due to noise adds undesired intra-variability in the ECG signals, creating the need for more robust biometric systems (BSs). With the increase of interest in the application of Deep Neural Networks (DNN) to the medical field, new solutions are also being explored in the identification and authentication of individuals. The proposed architecture exploits the potential of Convolutional Neural Networks (CNN) to identify healthy subjects using temporal frequency analysis, i.e. spectrograms.
\end{abstract}

Index Terms-Biometrics, electrocardiogram, deep learning, convolutional neural networks, spectrogram.

\section{INTRODUCTION}

The measurement of individual physical and/or behavioral characteristics may be used for biometric purposes. Biometric Systems (BSs) are present in our daily life, either for identification (e.g. identification of a fingerprint in a crime scene) or authentication (e.g. passport verification at an airport) of a person. In the last decade, with the increase in concerns about security, more trustworthy technologies are demanded by the military, government, and health sectors [1], [2].

There are several aspects that a BS should follow, such as universality, uniqueness, permanence, measurability, performance, acceptability and circumvention. The biometric signal may be classified in two areas: physical (e.g. fingerprint, biosignals), and behavioral (e.g. keystrokes, gait) [3].

The Electrocardiogram (ECG) has the potential to be chosen as the only source of information to a BS because it excels in critical aspects, such as: universality, everyone possesses a heart; uniqueness, the physical and chemical structural differences of the heart provide differences in electrical conduction; and circumvention, as it is extremely difficult to counterfeit these signals [2].

In the last two years, there was an increase in research on the biometric applications of Deep Neural Networks (DNN). This includes many successful approaches to the recognition of ECG signals, suggesting its usefulness for this task [4], [5]. Some of these approaches included the use of Convolutional Neural Networks (CNN), which are known for their success

Manuscript received February 12, 2019; revised December 5, 2019.

All authors are with LIBPhys (Laboratory for Instrumentation, Biomedical Engineering and Radiation Physics), Faculdade de Ciências e Tecnologia, Universidade Nova de Lisboa (e-mail: n.bento@campus.fct.unl.pt, \{dj.belo,hgamboa\}@fct.unl.pt). in the fields of image recognition and natural language processing [6]. Since the introduction of this algorithm, many improvements have been made. A relevant example is the DenseNet [7], a recently proposed CNN architecture which includes extra connections in groups of successive layers dense blocks.

This work takes advantage of these recent developments to conceive a biometric identification system based on ECG spectrograms and CNN. Our objective is to improve the accuracy and robustness of the current systems without the need to extract the ECG cycle. In order to evaluate the results, this method will be validated in two different databases, Fantasia [8] and ECG-ID [9], both acquired from PhysioNet [10].

This paper will guide through a brief explanation of ECG biometrics in Section II, followed by the state-of-the-art in biometrics (Section III). The used data is described in Section IV. Section V contains the signal processing steps and the architecture of the used network (CNN). The results, concerning the application of the proposed method on the two mentioned databases, are displayed in Section VI, while a discussion of the conclusions and future work to be made is presented in Section VII.

\section{ECG BIOMETRICS}

The ECG is a recording of an electrical signal which represents the activation of the heart muscles to trigger their movements. Each signal is represented by different waves: (1) $\mathrm{P}$ wave: Contraction of the atria; (2) PQ segment: Time between the contraction of the atria and activation of the ventricles; (3) QRS complex: The combination of the Q, R and $\mathrm{S}$ waves, associated with the contraction of the ventricles; (4) ST segment: Stage in which the ventricles contract; (5) T wave: The repolarization of the ventricles [2], [4].

Differences in shape and development of the muscles for different individuals change the electrical propagation and, consequently, the signal morphology. This inter-variability permits the identification of the ECG source for biometric purposes. There are two kinds of BSs, fiducial and non-fiducial based. The first relies on features extracted between reference points in the cardiac cycle (e.g. temporal intervals). As for the non-fiducial-based BSs, the ECG is considered as a whole, considering characteristics as R-R intervals or relative heights between waves [2], [11].

BSs that rely on biosignals often involve issues regarding the acquisition in terms of noise and artifact contamination, increasing the intra-subject variability. Consequently, computing features may be arduous as the electrode material, sensor location, movement, instrumentation of the devices, and power-line may compromise the match between ECG 
signals from the same source [12], [13].

BSs have two temporal phases, the enrolment, which requires an acquisition of the signal to make a template of the person and store it in a database, and the authentication/identification, when the same feature extraction methods are applied to the new acquired data and a match is given when comparing it with templates from the same database.

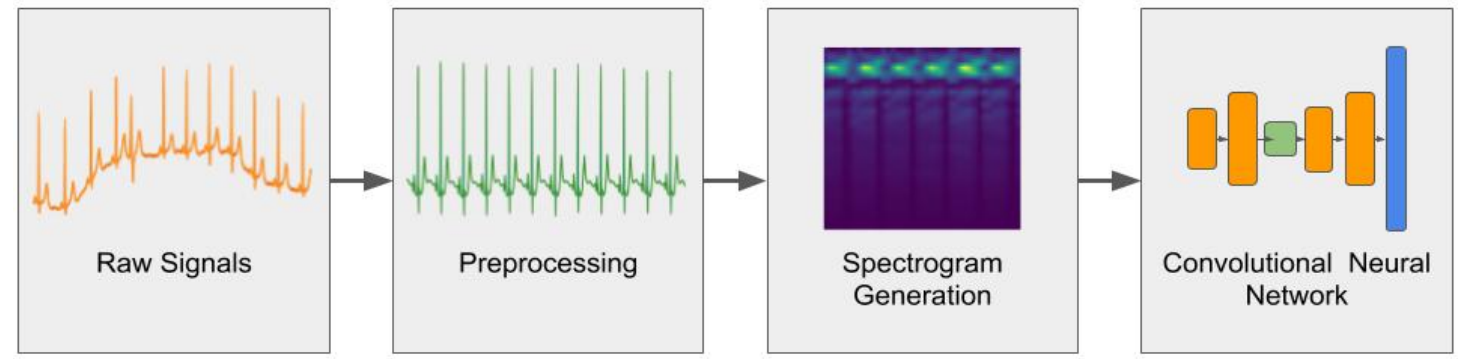

Fig. 1. Flowchart of the method.

The typical BS with ECG comprises the following flow: first, the acquired data is submitted to a feature extraction algorithm; then, the extracted features are fed to a classification module that compares the features to make a decision; finally, the features and/or classifier modules are stored in a database during the enrolment phase [14].

\section{RELATED WORK}

In ECG Biometrics research, the most common fiducial feature extraction methods identify morphological characteristic points [15] or resort to wavelet transform [16], discrete cosine transform [17] or Fourier transform to extract the cycle representation coefficients [18]. The resultant features may be submitted to a dimensional reduction method, such as Principal Component Analysis (PCA), Linear Discriminant Analysis (LDA) [19] or Dynamic Time Warping [20]. These methods may also be incorporated in the classification module [21], [22]. For non-fiducial approaches, R-R intervals and PCA are commonly used for feature extraction [22] . Ferdinando et al. [23] used a k-nearest neighbor $(\mathrm{kNN})$ classifier applied to bivariate empirical mode decomposition for biometric identification when emotions are considered. Besides achieving high classification rates, this paper found how the accuracy is affected by two spectrogram parameters: overlap percentage and window size.

DNNs have been employed in ECG biometrics to improve performance rates. Page et al. [24] used Neural Networks (NNs) for both QRS detection and classification, resulting in an accuracy of $99.96 \%$ for ECG-ID database. Eduardo et al. [25] implemented a Deep Autoencoder to learn lower dimensional feature representations, achieving low identification errors on a private dataset. Zheng et al. (2017) also used an Autoencoder to achieve 98.1\% accuracy using a self-collected database.

Salloum \& Kuo [26] proposed an aggregation of Recurrent Neural Networks (RNN) architectures for analysis and classification after selecting QRS segments without further feature extraction methods. For the identification problem, it was reported nearly $100 \%$ classification accuracy using the ECG-ID database. Zhang et al. [27] used a multiresolution one-dimensional $\mathrm{CNN}$, where the feature extraction step comprises discrete wavelet transform, autocorrelation and component selection, to obtain $97.2 \%$ identification rate for the Fantasia database. Luz et al. [5] used an architecture that fuses a $1 \mathrm{D} \mathrm{CNN}$, fed with raw ECG heartbeats, with another $\mathrm{CNN}$ with two dimensions, fed with the corresponding spectrograms, reaching state of the art performance on off-the-person ECG biometrics.

Spectrograms and CNNs have also been applied to ECG data in other settings, such as the detection of atrial fibrillation [28].

\section{DATA}

The data used in this paper is from two well-known public databases from PhysioNet database [10] used extensively in ECG biometrics studies: Fantasia and ECG-ID.

The Fantasia database consists on 40 subjects (20 young and 20 elderly) whose signals were recorded during a single 120 min session with a sampling frequency of $250 \mathrm{~Hz}$, while watching the movie Fantasia from Disney [8].

ECG-ID database consists of 310 recordings of 90 subjects aged from 13 to 75 . While the original data ranges from 2 to 20 recordings per subject, only the first two were used, with the purpose of having the same number of samples for each subject and ensuring a balanced dataset. Each recording is 20 seconds long with a sampling frequency of $500 \mathrm{~Hz}$ [9].
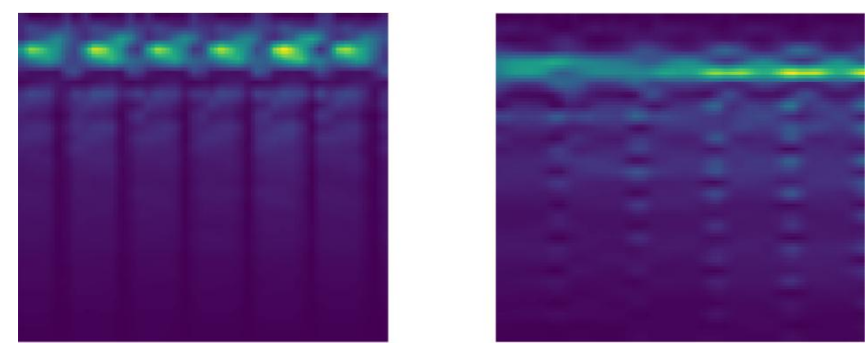

Fig. 2. Two examples of spectrograms for each used database: Fantasia (left) and ECG-ID (right).

\section{MEthods}

Our approach (Fig. 1) comprises 3 steps: preprocessing, spectrogram generation and subject identification $(\mathrm{CNN})$. These steps will be explained in detail.

\section{A. Signal Processing}

The signal preprocessing step is crucial in this work, as it makes the signals more interpretable to the neural network and reduces the noise from undesirable sources [12]. It is 
applied to the raw data, before performing the split between training and validation sets. First, each signal is scaled as follows:

$$
x^{\prime}=\frac{x-\bar{x}}{\max (\mathrm{x})-\min (\mathrm{x})}, \text { where } x \text { denotes the signal. }
$$

Then, a Hann window filter is applied, so that higher frequencies, which are associated with noise, are attenuated. Afterwards, a sliding window subtracts its moving average to the signal, removing baseline wandering.

\section{B. Spectrogram Generation}

A spectrogram is a visual representation of the signal magnitude at a frequency value through time, i.e. a frequency spectrum of a signal. That graphical representation comprises the $\mathrm{x}$-axis, time, the $\mathrm{y}$-axis, frequency, and the z-axis, the energy of each frequency at a given moment in time. The representation of the last may be seen as a surface in 3 dimensions or with different colors in 2 dimensions. In this work, the frequencies and correspondent magnitudes are obtained by the application of a Fourier transform to successive time windows resulting in a 2D matrix.

Since both databases have a significant difference in the recording duration, the need for a minimum amount of training and validation data requires a difference in the parameters used for spectrogram generation between databases.

For the Fantasia database, the signals are segmented into chunks with a length of 1536 samples (approximate duration of 6 seconds) and the fast Fourier transform window has a size of 256 samples with an overlap of $87.5 \%$. For the ECG-ID database, the segments consist in 2048 samples (approximately 4 seconds) and a fast Fourier transform window size of 512 , with an overlap of $93.75 \%$. The shorter duration of the segments is due to the need for increasing the number of generated spectrograms for training and validation. In both databases, all the segments are submitted to a Tukey window with an $\alpha$ parameter equal to 0.5 .

Once the spectrograms are generated, the frequencies above $120 \mathrm{~Hz}$ are removed, the resulting matrix is resized to $80 \times 80$ and scaled by subtracting the mean and dividing by the standard deviation. Examples of spectrograms are shown in Fig. 2.

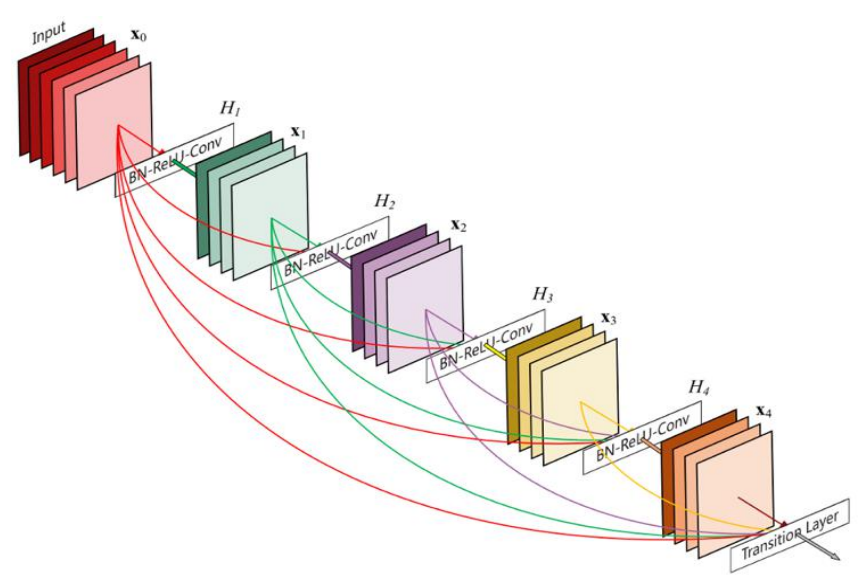

Fig. 3. "A 5-layer dense block with a growth rate of $\mathrm{k}=4$," reprinted from [7].

\section{Convolutional Neural Network}

The CNN is a type of NN that applies convolution operations between kernels and a tensor. In the case of image recognition, the image can either be provided as input to a $\mathrm{CNN}$ in the form of a 2-dimensional tensor (width and height), in case of having a single value that represents each pixel (grayscale), or as a 3-dimensional tensor (width, height, and number of channels), where the last dimension represents the components of each pixel. For example, the intensities of red, green, and blue are the three channels that can be used for RGB images.

The kernels can be interpreted as filters which detect shapes, edges, and other patterns that may appear in the image. These are composed by weights that can mutate as they learn, during the training process. In the forward propagation step, kernels are activated by passing through an activation function, a nonlinear function, such as the hyperbolic tangent or the rectified linear unit (ReLU). The latter is given by $\max (x, 0)$, being $x$ the input vector. It passes only values above zero, resembling its biological counterpart, the action potential. This feature allows the NN to solve nonlinear problems.

All the layers in a CNN that operate by convoluting kernels and tensors are called convolutional layers and their output is stack of two-dimensional feature maps.

After a convolution, a pooling layer can be used to reduce the dimensions of the feature maps. This procedure does not only minimize the computational effort, but also helps filters in the detection of shapes that are submitted to translations [6]. In this work, this operation consists on replacing consecutive patches of size $n \times n$ by either their maximum value (max pooling) or their average value (average pooling).

After the inputs are passed through a sequence of convolutional and pooling layers, in order to make it possible for the classification step to take place, the final outputs are flattened and can pass by one or more fully connected (regular) layers, until the class with the highest probability is assumed as the predicted class. The fully connected layers are simply composed of matrix multiplications between the inputs and the weights, with an optional addition of a bias parameter. The final activation is generally a softmax function, given by:

$$
\operatorname{softmax}\left(x_{i}\right)=\frac{e^{x_{i}}}{\sum_{j=0}^{K} e^{x_{j}}}, i=0,1,2, \ldots K,
$$

where $x$ is the output vector of the neural network.

After the initialization and the first forward pass, the network starts to learn. This learning step is done by iteratively propagating the partial derivative of the error with respect to the weights (i.e. backpropagation). The metric used to calculate the error is the cross entropy $(H)$ between the predicted values $(\hat{y})$ and the ground truth $(y)$, given by:

$$
H(y, \hat{y})=\sum_{i} y_{i} \log \left(\frac{1}{\hat{y}_{i}}\right)=-\sum_{i} y_{i} \log \left(\hat{y}_{i}\right) .
$$

It is possible to apply 2-dimensional CNNs to ECG data by changing the representation of the signals from a $1 \mathrm{D}$ vector of samples to a $2 \mathrm{D}$ matrix, using a spectrogram. This process adds the notion of frequency and makes the inputs interpretable to the network.

In our experiments, we compare the results of a simple CNN and a DenseNet [7]. In this type of architecture, the output of one layer is fed to all the following layers. 
Consequently, each dense block is the result of the concatenation of all the previous feature maps in that same set of layers (Fig. 3) and serves as input to the next layer. The number of feature maps in each layer inside a dense block is called growth rate. The transition layers, positioned between the flow of blocks, usually perform convolution and pooling operations. Besides allowing a more efficient use of the network parameters, this type of architecture achieved state of the art results on popular image recognition datasets.

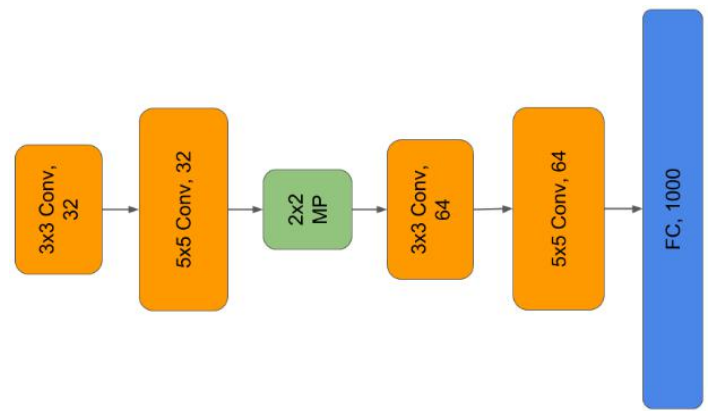

Fig. 4. CNN Architecture for Fantasia Database. "Conv", "MP", and "FC" respectively stand for convolutional, max pooling and fully connected layers.

\section{Architecture}

In this work, two different $\mathrm{CNN}$ architectures are compared. The first one is a simple CNN with 4 convolutional layers with ReLU activations, depicted in Fig. 4. This CNN does not only serve as a baseline for comparison with a larger network, but also to optimize the preprocessing parameters for both networks, reducing the required computational time. This optimization was made by an iterative process of checking which parameters for the construction of the spectrograms gave higher values of accuracy for subject identification. The second $\mathrm{CNN}$ is a DenseNet with 19 layers, including 3 dense blocks with 5 layers per block and a growth rate of 10 , starting with 20 kernels on each convolutional layer. The outputs of the last dense block are subsampled by a global average pooling operation. In theory, this network should learn more complex and diverse features, allowing better performance rates.

For both architectures, the output layer has a size which corresponds to the number of required classes and is passed through a softmax activation function.

TABLE I: Within-SESSION Classification PERFormance (\%)

\begin{tabular}{ccccc}
\hline \hline Model & Database & Accuracy & Sensitivity & Specificity \\
\hline $\begin{array}{c}\text { Simple } \\
\text { CNN }\end{array}$ & Fantasia & 99.42 & 99.42 & 99.98 \\
DenseNet & Fantasia & 99.79 & 99.78 & 99.99 \\
$\begin{array}{c}\text { Simple } \\
\text { CNN }\end{array}$ & ECG-ID & 94.23 & 94.26 & 99.94 \\
DenseNet & ECG-ID & 96.88 & 96.89 & 99.96 \\
\hline \hline
\end{tabular}

TABLE II: ECG-ID ACROSS-SESSION CLASSIFICATION PERFORMANCE (\%)

\begin{tabular}{cccc}
\hline \hline Model & Accuracy & Sensitivity & Specificity \\
\hline Simple & 73.54 & 72.72 & 99.70 \\
CNN & 73.28 & 72.46 & 99.70 \\
DenseNet & & & \\
\hline \hline
\end{tabular}

\section{RESULTS}

The proposed task is to classify ECG segments into as many classes as individuals present in the system. For these experiments, Adam [29] is chosen as the optimizer for both CNNs. The Keras package [30] is used for the implementation of the networks as a high-level wrapper for TensorFlow [31], a numerical computation library that allows parallel processing.

In the case of within-session experiments, the models are trained on $67 \%$ of the total length of the signals and validated on the remaining $33 \%$. For the Fantasia database, spectrograms are randomly chosen throughout the entire length of every signal, as the recording time is much longer when compared to ECG-ID, of which all the generated spectrograms were used. The total amount of spectrograms for Fantasia is 39166 for training and 19094 for testing, while for ECG-ID, the corresponding quantities are 25955 and 6981 , respectively.

In the case of the across-session experiments in ECG-ID, the second recording session of each individual was used for validation, while the first one was used as the training set. This generated 22320 spectrograms for training and 22072 for testing. All the remaining parameters were fixed.

Training was performed on two NVIDIA GeForce GTX 1080 Ti graphics cards.

The evaluation metrics were the following:

$$
\begin{aligned}
\text { Accuracy }= & (T P+T N) /(T P+F P+T N+F N), \\
\text { Sensitivity }= & \frac{T P}{T P+F N}, \\
\text { Specificity } & =\frac{T N}{T N+F P},
\end{aligned}
$$

where $T P, T N, F P$, and $F N$ respectively stand for True Positives, True Negatives, False Positives, and False Negatives.

TABLE III: ACCURACY COMPARISON FOR FANTASIA DATABASE. RBF AND RF STAND, RESPECTIVELY, FOR RADIAL BASIS FUNCTION AND RANDOM

\begin{tabular}{ccc}
\multicolumn{3}{c}{ FOREST } \\
\hline \hline Work & Method & Accuracy (\%) \\
\hline$[16]$ & Wavelets and RBF NN & 95.89 \\
{$[33]$} & Hand-crafted features and RF & 98 \\
{$[27]$} & Wavelets and 1D CNN & 97.2 \\
\hline Proposed & Spectrograms and Small CNN & 99.42 \\
Proposed & Spectrograms and DenseNet & 99.79 \\
\hline \hline
\end{tabular}

TABLE IV: WITHIN-SESSION ACCURACY COMPARISON FOR ECG-ID DATABASE. PCA, LDA, AND (W)NM STAND, RESPECTIVELY, FOR PRINCIPAL COMPONENT ANALYSIS, LINEAR DISCRIMINANT ANALYSIS AND (WEIGHTED) NEAREST MEAN

\begin{tabular}{ccc}
\hline \hline Work & Method & Accuracy (\%) \\
\hline$[9]$ & Wavelets, PCA and LDA+(W)NM & 96 \\
ensemble & Wavelets and Probabilistic RF & 98.79 \\
{$[32]$} & NNs for QRS detection and & 99.96 \\
{$[24]$} & classification & 98.2 \\
{$[26]$} & LSTM (3 beats as input) & 100 \\
{$[26]$} & LSTM (9 beats as input) & 94.23 \\
\hline Proposed & Spectrograms and Small CNN & 96.88 \\
Proposed & Spectrograms and DenseNet & \\
\hline \hline
\end{tabular}

The performance of the proposed method reaches state of the art on both Fantasia and ECG-ID databases, being the values of sensitivity and specificity (Table I) within a 
reasonable range.

The results presented on Table III suggest that the combination of deep learning and spectrograms can be used effectively for human identification using a non-fiducial approach, only needing a recording of 6 seconds to be able to accurately classify a subject on a universe of 40 .

As for the ECG-ID database, the used bibliography for comparison outlined in Table IV was: Lugovaya [9], Tan \& Perkowski [32], Page et al. [24] and Salloum \& Kuo [26]. These papers diverged in the selection of data for cross-validation, as it ranges from 2 to 20 sessions per subject. The first three use all the available data, while the latter only used a single session per subject. In our work, the characterization of this dataset uses 2 sessions per subject and 4 second segments, balancing the training data for each individual, close to the standard of fingerprint authentication databases.

TABLE V: ACROSS-SESSION ACCURACY COMPARISON FOR ECG-ID DATABASE

\begin{tabular}{ccc}
\hline \hline Work & Method & Accuracy (\%) \\
\hline$[26]$ & LSTM (3 beats as input) & 97 \\
{$[26]$} & LSTM (9 beats as input) & 100 \\
\hline Proposed & Spectrograms and Small CNN & 73.54 \\
Proposed & Spectrograms and DenseNet & 73.28 \\
\hline \hline
\end{tabular}

In the across-session experiments (Table V), both models reach similar results. This may have to do with the fact that these models were overfitting to a small training set (10s) with a very high overlap percentage (93.75\%), which generated very similar spectrograms, not enabling the models to learn some of the features required to better distinguish tween subjects.

Even though our results for both architectures are not as accurate on ECG-ID database as on Fantasia (Table III), the accuracy is still high, offering a good basis for this preliminary work. This could be attributed to several factors. The first one may have occurred as a result of the variability in the selection of data, which can be compromising the comparison. The second is due to the larger number of subjects, as the increase in the number of classes leads to lower accuracy values for similar models. At last, the short recording time, only allowing the generation of 366 spectrograms per subject, even with a considerable overlap percentage (93.75\%). This jeopardizes the training, as the CNN requires a high amount of data to achieve good performance. These limitations are opportunities to optimize our method.

\section{CONCLUSION AND FUTURE WORK}

Through the recognition of temporal and frequential information existent in spectrograms, we were able to confidently attribute small segments of ECG signals to the subject from whom they were created. This represents another step towards more capable and robust biometric identification/authentication systems.

One of the advantages of this algorithm is its robustness to variations in the moment of signal acquisition, as spectrograms with small offsets in time were correctly identified without the need for QRS detection.
Although the achieved results showed good performance, there is still a considerable margin for improvement. The use of external data for pretraining could be helpful, as it may enable the network to learn a better latent representation of the signal, especially in low data regimes, as is the case of the ECG-ID database. This would likely improve its generalization capability, allowing the system to differentiate between more subjects and require less training samples for each one. Further development could also be made regarding signal acquisition and preprocessing, as the effect of noise and artifacts present in the signal is a probable cause for recognition error.

While this paper demonstrates there is potential in the use of spectrograms for biometric recognition applications, future work should include testing in data acquired in different periods, separated by several months or years, in order to prove the reliability of these systems over time.

\section{CONFLICT OF INTEREST}

The authors declare no conflict of interest.

\section{AUTHOR CONTRIBUTIONS}

Nuno Bento and David Belo conducted the research and wrote the paper; Hugo Gamboa supervised the work and reviewed the paper; all authors had approved the final version.

\section{ACKNOWLEDGMENT}

The authors thank Nova University of Lisbon and Fundação para a Ciência e Técnologia for the given support.

\section{REFERENCES}

[1] J. Unar, W. Seng, and A. Abbasi, "A review of biometric technology along with trends and prospects," Pattern Recognition, vol. 47, no. 8, pp. 2673-2688, 2014.

[2] A. Nait-Ali, "Hidden biometrics: Towards using biosignals and biomedical images for security applications," in Proc. 7th international Workshop on Systems, Signal Processing and Their Applications, 2011, pp. 352-356.

[3] T. Araújo, N. Nunes, H. Gamboa, and A. Fred, "Generic biometry algorithm based on signal morphology information: Application in the electrocardiogram signal," in Proc. International Conference on Pattern Recognition Applications and Methods, 2015, pp. 301-310.

[4] D. Belo, J. Rodrigues, J. R. Vaz, P. Pezarat-Correia, and H. Gamboa, "Biosignals learning and synthesis using deep neural networks," Biomed. Eng. Online, vol. 16, no. 1, p. 115, 2017.

[5] E. Luz, G. Moreira, L. S. Oliveira, W. Schwartz, and D. Menotti, "Learning deep off-the-person heart biometrics representations," IEEE Transactions on Information Forensics and Security, 2017.

[6] I. Goodfellow and A. Courville, Deep Learning, 2016.

[7] G. Huang, Z. Liu, L. Maaten, and K. Q. Weinberger, Densely Connected Convolutional Networks, vol. 1, no. 2, p. 3, 2017.

[8] N. Iyengar, C. Peng, R. Morin, A. L. Goldberger, and L. A. Lipsitz, "Age-related alterations in the fractal scaling of cardiac interbeat interval dynamics," American Journal of Physiology-Regulatory, Integrative and Comparative Physiology, vol. 271, no. 4, pp. R1078-R1084, 1996.

[9] T. Lugovaya, "Biometric human identification based on electrocardiogram," LETI, 2005.

[10] A. L. Goldberger et al., "Physiobank, physiotoolkit, and physionet," Circulation, vol. 101, no. 23, pp. e215-e220, 2000.

[11] F. Gargiulo, A. Fratini, M. Sansone, and C. Sansone, "Subject identification via ECG fiducial-based systems: Influence of the type of QT interval correction," Comput Methods Programs Biomed., vol. 121, no. 3, pp. 127-136, Elsevier Ireland Ltd, 2015.

[12] J. Rodrigues, D. Belo, and H. Gamboa, "Noise detection on ECG based on agglomerative clustering of morphological features," Computers in Biology and Medicine, vol. 87, pp. 322-334, 2017. 
[13] S. Fang and H. Chan, "QRS detection-free electrocardiogram biometrics in the reconstructed phase space," Pattern Recogn. Lett., vol. 34, no. 5, pp. 595-602, 2013.

[14] E. L. Broek, Beyond Biometrics, vol. 1, no. 1, pp. 2511-2519, 2010.

[15] L. Biel, O. Pettersson, L. Philipson, and P. Wide, "ECG analysis: a new approach in human identification," IEEE Transactions on Instrumentation and Measurement, vol. 50, no. 3, pp. 808-812, 2001.

[16] M. Tantawi, K. Revett, A.-D. Salem, and M. F. Tolba. (2013). ECG based biometric recognition using wavelets and RBF neural network [Online]. Available: https://www.semanticscholar.org/paper/ECG-based-Biometric-Recog nition-using-Wavelets-and-Tantawi-Revett/d01999b97953b12a1c124 0b796bc74715f2ccb62

[17] Y. Wang, F. Agrafioti, D. Hatzinakos, and K. N. Plataniotis, "Analysis of human electrocardiogram for biometric recognition," EURASIP Journal on Advances in Signal Processing, vol. 2008, no. 1, p. 148658, 2007.

[18] S. Saechia, J. Koseeyaporn, and P. Wardkein, Human identification System Based ECG Signal, pp. 1-4, 2005.

[19] M. Tantawi, K. Revett, A. Salem, and M. Tolba, "Fiducial feature reduction analysis for electrocardiogram (ECG) based biometric recognition," Internat. J. Intell. Inf. Sys., vol. 40, no. 1, pp. 17-39, 2013.

[20] J. Shen, S. D. Bao, L. C. Yang, and Y. Li, "The PLR-DTW method for ECG based biometric identification," in Proc. 2011 Annual International Conference of the IEEE Engineering in Medicine and Biology Society, 2011, pp. 5248-5251.

[21] S. A. Israel, J. M. Irvine, A. Cheng, M. D. Wiederhold, and B. K. Wiederhold, "ECG to identify individuals," Pattern Recognition, vol. 38, no. 1, pp. 133-142, 2005.

[22] I. A. Chamatidis and G. Spathoulas, "Using deep learning neura networks for ECG based authentication," in Proc. 2017 Int. Carnahan Conf. Secur. Technology Iccst, 2017, pp. 1-6.

[23] H. Ferdinando, T. Seppänen, and E. Alasaarela, "Bivariate empirical mode decomposition for ECG-based biometric identification with emotional data," in Proc. IEEE Conf. on Eng. Med. Biol. Soc., 2017, pp. $450-453$

[24] A. Page, A. Kulkarni, and T. Mohsenin, "Utilizing deep neural nets for an embedded ECG-based biometric authentication system," in Proc. 2015 IEEE Biomedical Circuits and Systems Conference, 2015, pp. $1-4$.

[25] A. Eduardo, H. Aidos, and A. Fred, "ECG-based biometrics using a deep autoencoder for feature learning," in Proc. ICPRAM, 2017.

[26] R. Salloum and C.-C. Kuo, "ECG-based biometrics using recurrent neural networks," in Proc. IEEE international conference on Acoustics, Speech and Signal Processing, 2017, pp. 2062-2066.

[27] Q. Zhang, D. Zhou, and X. Zeng, "HeartID: A multiresolution convolutional neural network for ECG-based biometric human identification in smart health applications," IEEE Access, 2017.

[28] M. Zihlmann, D. Perekrestenko, and M. Tschannen, "Convolutional recurrent neural networks for electrocardiogram classification," Computing, vol. 44, p. 1, 2017.

[29] D. Kingma and J. Ba, "Adam: A method for stochastic optimization," arXiv preprint arXiv: 1412.6980, 2014.

[30] F. Chollet. (2015). Keras. GitHub Repository. [Online]. Available: https://github.com/fchollet/keras
[31] M. Abadi et al. (2015). TensorFlow: Large-Scale Machine Learning on Heterogeneous Systems. [Online]. Available: https://www.tensorflow.org

[32] R. Tan and M. Perkowski, "ECG Biometric Identification Using Wavelet Analysis Coupled with Probabilistic Random Forest," in Proc. 2016 15th IEEE International Conference on Machine Learning and Applications, 2016, pp. 182-187.

[33] Q. Zhang, D. Zhou, and X. Zeng, "Machine learning-empowered biometric methods for biomedicine applications," AIMS Medical Science, vol. 4, pp. 274-290, 2017.

Copyright (C) 2020 by the authors. This is an open access article distributed under the Creative Commons Attribution License which permits unrestricted use, distribution, and reproduction in any medium, provided the original work is properly cited (CC BY 4.0).

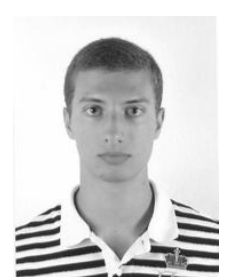

Nuno Bento was born in Beja, Portugal on November 12, 1994. He is a masters student in biomedical engineering at Faculdade de Ciências e Tecnologia Universidade Nova de Lisboa.

From 2017 to 2018, he did an internship at Laboratory for Instrumentation, Biomedical Engineering and Radiation Physics - UNL, contributing to research in deep learning. His research interests include machine learning and biomedical signa processing, with applications in the medical sector and in biometrics-based security systems.

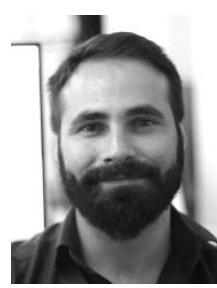

David Belo was born in Lisbon, Portugal on October 1, 1983. He received a Master Degree in Biomedical Engineering at University of Minho in 2007.

$\mathrm{He}$ has experience in electroencephalograms, electrocardiograms and machine learning. He alsohas experience in programming and teaching outside the academics. He is currently a researcher and a $\mathrm{PhD}$ student at in Nova University of Lisbon, researcher a LIBPHYS-UNL in the area of Deep Neural Networks and is producing work in synthesis and classification of biosignals.

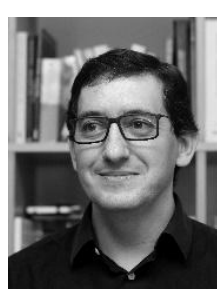

Hugo Gamboa received a $\mathrm{PhD}$ in electrical and computer engineering from Instituto Superior Técnico, Technical University of Lisbon. His thesis entitled 'Multi-modal behavioral biometrics based on HCI and electrophysiology'. He is a founder and president of PLUX, a technology-based innovative company in the field of systems and wireless medical sensors, where he currently coordinates the scientific direction of a multidisciplinary team that covers microelectronics, biosignal processing and software development. He has a notorious work on medical instrumentation, auxiliar professor in Nova University of Lisbon, researcher at LIBPHYS-UNL and senior scientist at Fraunhofer Portugal. 